\title{
Associations between urbanicity and malaria at local scales in Uganda
}

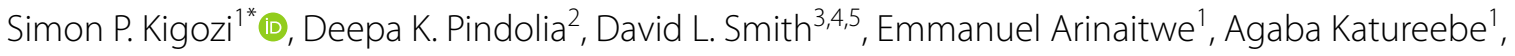
Maxwell Kilama', Joaniter Nankabirwa' ${ }^{1}$ Steve W. Lindsay ${ }^{6}$, Sarah G. Staedke', Grant Dorsey ${ }^{8}$, Moses R. Kamya ${ }^{1,9}$ and Andrew J. Tatem $3,10,11$

\begin{abstract}
Background: Sub-Saharan Africa is expected to show the greatest rates of urbanization over the next 50 years. Urbanization has shown a substantial impact in reducing malaria transmission due to multiple factors, including unfavourable habitats for Anopheles mosquitoes, generally healthier human populations, better access to healthcare, and higher housing standards. Statistical relationships have been explored at global and local scales, but generally only examining the effects of urbanization on single malaria metrics. In this study, associations between multiple measures of urbanization and a variety of malaria metrics were estimated at local scales.

Methods: Cohorts of children and adults from 100 households across each of three contrasting sub-counties of Uganda (Walukuba, Nagongera and Kihihi) were followed for 24 months. Measures of urbanicity included density of surrounding households, vegetation index, satellite-derived night-time lights, land cover, and a composite urbanicity score. Malaria metrics included the household density of mosquitoes (number of female Anopheles mosquitoes captured), parasite prevalence and malaria incidence. Associations between measures of urbanicity and malaria metrics were made using negative binomial and logistic regression models.

Results: One site (Walukuba) had significantly higher urbanicity measures compared to the two rural sites. In Walukuba, all individual measures of higher urbanicity were significantly associated with a lower household density of mosquitoes. The higher composite urbanicity score in Walukuba was also associated with a lower household density of mosquitoes (incidence rate ratio $=0.28,95 \% \mathrm{Cl} 0.17-0.48, \mathrm{p}<0.001$ ) and a lower parasite prevalence (odds ratio, $\mathrm{OR}=0.44, \mathrm{Cl} 0.20-0.97, \mathrm{p}=0.04$ ). In one rural site (Kihihi), only a higher density of surrounding households was associated with a lower parasite prevalence $(\mathrm{OR}=0.15, \mathrm{Cl} 0.07-0.34, \mathrm{p}<0.001)$. And, in only one rural site (Nagongera) was living where $\mathrm{NDVI} \leq 0.45$ associated with higher incidence of malaria (IRR $=1.35, \mathrm{Cl} 1.35-1.70, \mathrm{p}=0.01)$.

Conclusions: Urbanicity has been shown previously to lead to a reduction in malaria transmission at large spatial scales. At finer scales, individual household measures of higher urbanicity were associated with lower mosquito densities and parasite prevalence only in the site that was generally characterized as being urban. The approaches outlined here can help better characterize urbanicity at the household level and improve targeting of control interventions.
\end{abstract}

Keywords: Urbanization, Plasmodium falciparum, Remote sensing, GIS, Urban malaria

\section{Background}

Urbanization has generally been associated with a reduction in malaria transmission across spatial scales [1-5]. Lower transmission of both Plasmodium falciparum [5]

\footnotetext{
*Correspondence: skigozi@yahoo.com

${ }^{1}$ Infectious Diseases Research Collaboration, Kampala, Uganda

Full list of author information is available at the end of the article
}

and Plasmodium vivax [3] has been found consistently in urban areas, compared to surrounding areas and multiple factors contribute to this. Urban areas generally have fewer mosquito breeding sites, better access to treatment and higher intervention coverage levels than rural areas [6, 7]. Urbanization also involves significant ecological change and socio-economic change, including improved health, housing and wealth factors that impel significant

\section{Biomed Central}

(c) 2015 Kigozi et al. This article is distributed under the terms of the Creative Commons Attribution 4.0 International License (http://creativecommons.org/licenses/by/4.0/), which permits unrestricted use, distribution, and reproduction in any medium, provided you give appropriate credit to the original author(s) and the source, provide a link to the Creative Commons license, and indicate if changes were made. The Creative Commons Public Domain Dedication waiver (http://creativecommons.org/ publicdomain/zero/1.0/) applies to the data made available in this article, unless otherwise stated. 
entomological, parasitological and behavioural effects that generally reduce malaria transmission $[2,4,5]$ and many malaria-endemic nations have recorded reduced malaria transmission in major cities [2, 8]. Previous studies on the impact of 'urbanicity' have generally looked at malaria parasite prevalence over large scales or at specific metrics at small scales, and using a single indicator of urbanicity [9]. No studies have examined a full range of entomological, parasitological and clinical-based metrics across multiple transmission sites and using multiple metrics of urbanicity.

Malaria remains a major problem in Uganda and many efforts have been made to control it including, but not limited to, provision and/or promotion of long-lasting insecticidal nets (LLINs) and indoor residual spraying of insecticides (IRS) [10]. Interventions to date have predominantly targeted vulnerable populations such as expectant mothers and children, and been implemented uniformly across wide geographic areas. However, approaches that target interventions to local conditions could improve efficiency and impact in settings where resources are limited. Aside from the targeted implementation of interventions, there is a need to better predict the effects that trends in urbanization, climate change, land use and development are likely to have on the burden of malaria in Uganda and other African countries. Most of Uganda's population live in relatively high-transmission areas [11] and despite recent expansion of control efforts, evidence of a clear reduction in the burden of disease is lacking $[12,13]$. A better understanding of the relationship between urbanicity and malaria risks at various scales may help to improve the targeting of strategies for malaria control, such as the initiation of integrated vector management (IVM) where larval source management (LSM) may be the chosen approach for urban locales, and IRS the approach for the rural, less densely populated areas [14]. Urbanization is a highly relevant topic for planning in Uganda because, although the populations are currently predominantly rural, high rates of population growth are leading to rapid urbanization.

Here, existing cohort studies were utilized in an exploratory analysis to examine associations between various measures of urbanicity and entomological, parasitological and clinical malaria metrics. The studies were conducted in three sites in Uganda with different levels of urbanicity and transmission intensity. Because of the underlying differences in the sites, several physical and infrastructure-based urbanicity metrics were evaluated that had consistent and quantifiable spatial characteristics, including the densities of surrounding households from survey data, and satellite image variables covering land use, night-time lights and occurrence or amount of vegetation.

\section{Methods \\ Study sites}

Cohort studies were conducted at three sites in Uganda:(1) Walukuba sub-county in Jinja district $\approx 12.9 \mathrm{sq} \mathrm{km}\left(00^{\circ} 26^{\prime} 33 \cdot 2^{\prime \prime} \mathrm{N}, 33^{\circ} 13^{\prime} 32 \cdot 3^{\prime \prime} \mathrm{E}\right)$ at the shores of Lake Victoria; (2) Kihihi sub-county in Kanungu district $\approx 186 \mathrm{sq} \mathrm{km}\left(00^{\circ} 45^{\prime} 03 \cdot 1^{\prime \prime} \mathrm{S}, 29^{\circ} 42^{\prime} 03.6^{\prime \prime} \mathrm{E}\right)$ in the southwest part of Uganda; and, (3) Nagongera subcounty in Tororo district $\approx 81 \mathrm{sq} \mathrm{km}\left(00^{\circ} 46^{\prime} 10 \cdot 6^{\prime \prime} \mathrm{N}\right.$, $34^{\circ} 01^{\prime} 34 \cdot 1^{\prime \prime} \mathrm{E}$ ) close to the Uganda-Kenya border. Figure 1 shows the location of the sites. Clinical activities were conducted at the respective health sub-district level IV health centres.

Jinja district was historically the industrial capital of Uganda and as such, Walukuba was urbanized in the industrial times of 1950-80s, hence the presence of factory buildings (now deserted for the most part), factory worker estates, more tarmac roads, and wider coverage of hydro-electric power than other sites. In Nagongera, according to a 2009/2010 survey, $2.6 \%$ of households had electricity, $59.8 \%$ of the homes were grass thatched [15], and less than half a kilometre of tarmac road was observed. Kihihi has similar characteristics to Nagongera, with almost no tarmac road (less than $3 \mathrm{~km}$ ), similar housing structures (walls of mud and poles) and its sources of water predominantly being open wells, bore holes and rainwater harvesting.

\section{Participant recruitment and follow-up}

All households in the three sub-counties were enumerated in a census survey and geo-located using a Garmin e-Trex Legend H GPS unit (Garmin International, Inc. Olathe, KS, USA). A household was defined as any single permanent or semi-permanent dwelling structure for humans who generally cook and/or eat together. One hundred households were randomly selected from each of the sub-counties and all children aged 6 months to 10 years and one adult primary caregiver from each household were enrolled as previously described [16]. At enrolment a survey was performed for each household. Figure 1 shows the distribution of households that were included in the cohort studies.

The cohorts were followed from August 2011 to September 2013, and they were dynamic, such that all newly eligible children were enrolled and study participants who reached 11 years of age were excluded from further follow-up. At enrolment, study participants and their parents/guardians were given a LLIN and underwent a standardized evaluation, including a history, physical examination and collection of blood for haemoglobin measurement and thick/thin blood smear. Cohort study participants received all medical care free of charge at a designated study clinic open every day. Participants who 


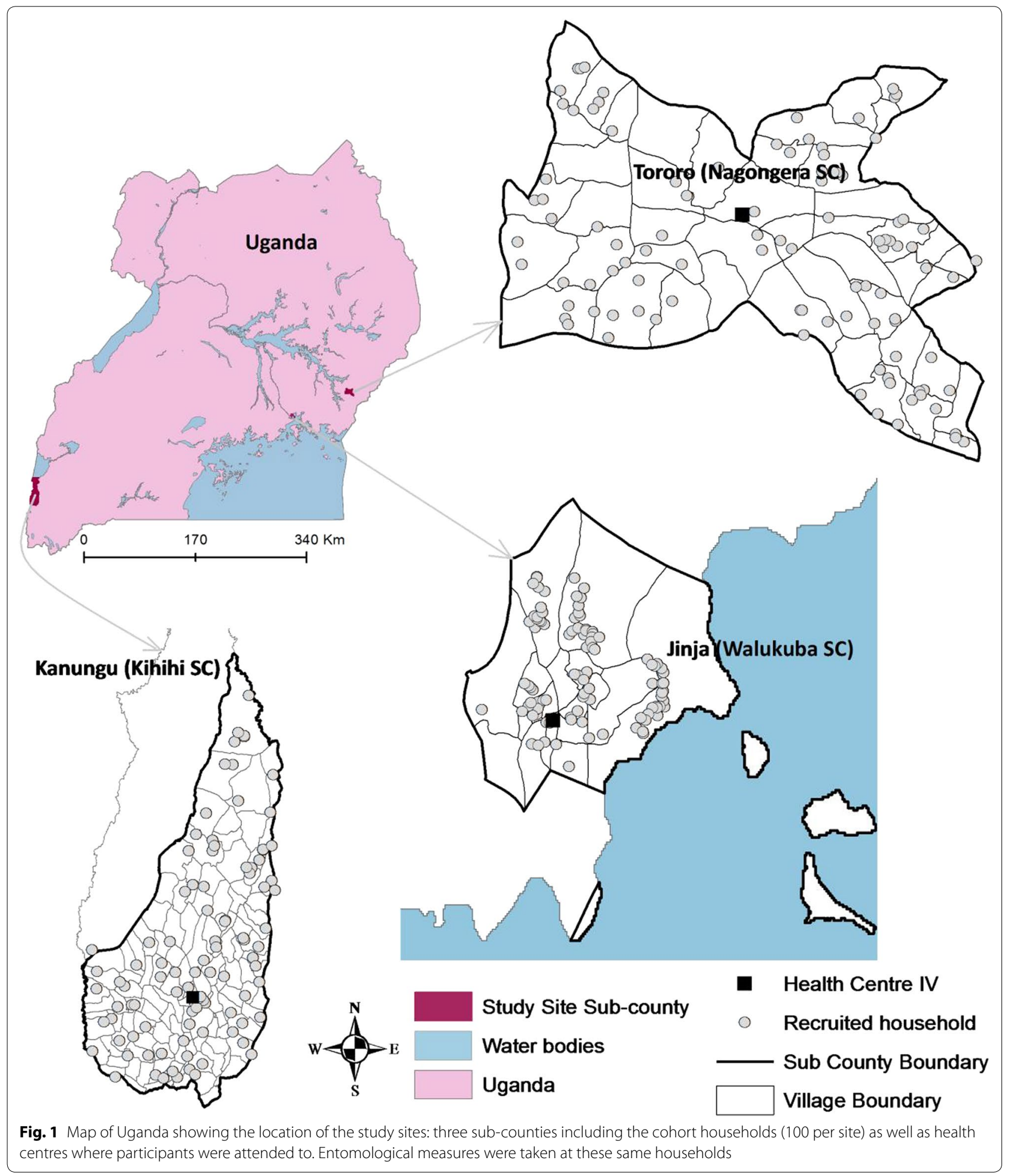

presented with a documented fever (tympanic temperature $>38.0{ }^{\circ} \mathrm{C}$ ) or history of fever in the previous $24 \mathrm{~h}$ had blood obtained by finger prick for a thick blood smear. If the smear had malaria parasites, the patient was diagnosed with malaria. Episodes of uncomplicated malaria were treated with artemether-lumefantrine and episodes of complicated malaria or recurrent malaria occurring within 14 days of prior therapy were treated 
with quinine. Routine thick blood smears were done every 3 months to estimate parasite prevalence.

Entomological surveys were conducted monthly from August 2011 to September 2013 in each household using miniature CDC light traps (Model 512; John W. Hock Company, Gainesville, FL, USA) with the light positioned $1 \mathrm{~m}$ above the floor at the foot end of the bed where a cohort study participant slept. Traps were set at $19.00 \mathrm{~h}$ and collected at 07.00 the following morning by field workers. Methods used for the processing of mosquito specimens were as described previously [17].

\section{Malaria metrics}

Malaria metrics used in this study included the household density of mosquitoes (HDM), parasite prevalence by light microscopy, and the incidence of malaria. The HDM was calculated as the total number of female Anopheles mosquitoes captured divided by the number of housenights of collection. Parasite prevalence was calculated as the number of routine blood smears positive for asexual parasites divided by the total number of routine blood smears done. The incidence of malaria was calculated as the number of episodes of malaria divided by the person years of observation. The malaria metrics used here can be placed on a spectrum that is comprised of three major components: the transmission phase, at the frontline between environment and the human host; the infection phase, an indication of malaria parasites independent of symptoms; and, the clinical phase, which manifests itself as a documented infection in the setting of symptomatic illness. In this study, HDM is in the first phase of this spectrum while parasite prevalence is apportioned to the second phase, one level removed from the environment, and the incidence of malaria is apportioned to the third phase, two levels removed from the environment.

\section{Measures of urbanicity}

Many different methods have been used in previous studies to provide consistent measures of either binary urban-rural classifications, or continuous quantification of urbanicity. Urbanicity can be measured in multiple ways and in this study physical-based measures that could be consistently derived between and across sites were measured, including household density, land cover, vegetation amount, and night-time light brightness. For each cohort household, 100-m buffer zones, also known as observational buffers, were created for generating estimates of household level measures of urbanicity by extracting data using ESRI ArcGIS 10.1 software (ESRI 1995-2013; Redlands, CA, USA). Cohort households accounted for $0.8,1.0$ and $1.4 \%$ of the total number of households enumerated in the Kihihi, Walukuba, and Nagongera sub-counties, respectively.

\section{Household density}

Household density was defined as the number of households enumerated in the observational buffers around each cohort study household.

\section{Land cover}

A supervised classification of Landsat imagery was undertaken, using Google earth [18] to define training samples of known land cover type. Landsat Enhanced Thematic Mapped (ETM) images matching the period of data collection were obtained from the US geological survey repository [19]. Following previously defined approaches [20], the training samples were used within a maximum likelihood supervised classifier in the satellite image processing software, Erdas Imagine (Geosystems, L. 2004; ERDAS imagine. Atlanta, GA, USA), to produce a land cover map that contained classes representing different gradations of urbanicity (Fig. 2). Among the three sites, only Walukuba displayed distinctly observable urban classes. The others, Nagongera and Kihihi, did not display similar classes, and thus, classification results were not used here for those sites. Within Walukuba, three distinct 'urban' related classes existed: dense urban, medium dense urban and residential low dense urban (Fig. 2). The percentage of $30 \times 30$-m grid cells in the observational buffer in each of these classes was used as a household level metric of urbanicity to test against the malaria metrics. Only the residential low dense urban classification of land cover showed significant associations with malaria metrics and hence was maintained while the other two were dropped from further analysis.

\section{Normalized difference vegetation index (NDVI)}

NDVI is measured on a scale of -1 to 1 where the extreme negative values represent water; values around zero reflect barren earth surfaces such as rock, sand or snow; medium positive values represent bushes or grassland; and, the higher positive values $(\geq 0.6)$ represent temperate/tropical rainforest cover [21]. NDVI often forms the basis for mapping degrees of urbanicity in wellvegetated regions of the world, where urbanized regions are relatively lowly vegetated compared to surrounding rural areas. In this study, SPOT 6, 6-m resolution satellite imagery acquired over 2013 with at most $1 \%$ cloud cover (Astrium GEO-Information Services, 2013, South Africa) was used to generate NDVI.

\section{Night-time lights}

Urban areas generally display greater rates of electrification than rural ones [22, 23], and thereby the amount of lighting detected through night-time satellite imagery has been used to quantify both the presence of urban areas and degree of urbanicity $[2,3]$. In this study, imagery 

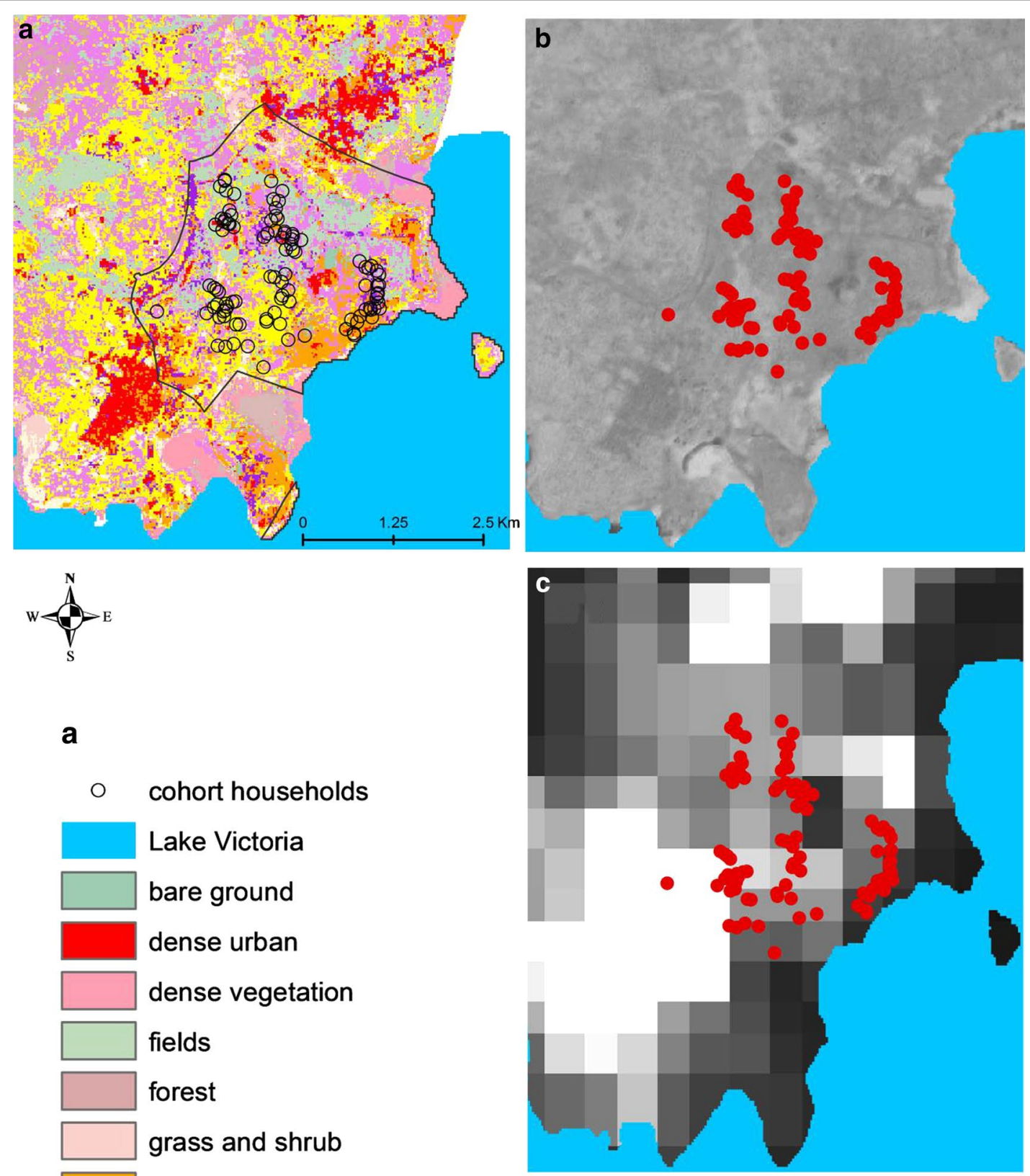

O cohort households

\begin{tabular}{|c|}
\hline Lake Victoria \\
\hline bare ground \\
\hline dense urban \\
\hline dense vegetation \\
\hline fields \\
\hline forest \\
\hline grass and shrub \\
\hline med-dense-urban \\
\hline mixed-residential-trees \\
\hline residential-lowdense \\
\hline road \\
\hline scrubland \\
\hline short-grass \\
\hline
\end{tabular}

b \& C

- cohort households

Lake Victoria

NDVI \& NTL

High

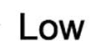

Fig. 2 Distribution of three urbanicity metrics across the three sites, based on the frequency distribution of individual metric values within a $100 \mathrm{~m}$ buffer around households participating in the cohort and entomology study in each site. a Land cover classification in Walukuba at $30 \mathrm{~m}$ spatial resolution overlaid with the participating study households. b NDVI in Walukuba at $6 \mathrm{~m}$ spatial resolution. c Satellite-derived night-time light brightness across Walukuba overlaid with the participating study households 
from the visible infrared imaging radiometer suite (VIIRS) satellite sensor was obtained for the three sites (from http://ngdc.noaa.gov/eog/viirs) and the levels of brightness within the observational buffers were estimated on a relative scale.

\section{Composite urbanicity score}

In addition to the individual urbanicity metrics measured in this study, overall urbanicity at each participating household, referred to as the composite urbanicity score or composite score, was estimated using the values of the individual urbanicity metrics in the observational buffer of the household. A score of 1 was assigned for every instance of a high level of individual urbanicity metric and 0 otherwise (Tables $1,2,3$ ), and the cumulative score, between 0 and 4, from the four individual urbanicity metrics was assigned as the composite score for the participating household.

\section{Statistical analyses}

Data collected were analysed using Stata 12 (StataCorp, TX, USA). Lowess smoothing plots were used to explore the relationships between continuous measures of urbanicity and the various malaria metrics. Based on these exploratory analyses, continuous measures of urbanicity for participating households were dichotomized into the following categories for estimating associations with malaria metrics: (1) household density ( $\leq 80$ vs $>80$ households per $100-\mathrm{m}$ radius buffer); (2) night-time lights ( $\leq 3.0$ vs $>3.0$ units of single band 32 bit pixels); (3) percentage residential low dense urban land cover $(\leq 20$ vs $>20 \%$ ); (4) NDVI ( $\leq 0.45$ vs $>0.45)$; (5) composite score (low versus high, see Tables 1, 2, 3). At the household level, negative binomial regression was used to model the relationships between measures of urbanicity and the HDM (number of female Anopheles caught per house), with the number of sampling nights included as an offset term in the model. At the individual cohort participant level, logistic regression was used to model the relationships between measures of urbanicity and the odds of malaria infection (parasite prevalence) at the time of each routine clinic visit, and negative binomial regression was used to model these relationships with the incidence of malaria, with the duration of follow-up included as an offset term. Analyses at the individual level were adjusted for age of the study participants and robust standard errors were used to adjust for clustering of cohort participants living in the same household. Associations using negative binomial regression models were expressed as the incidence rate ratio (IRR) and associations using logistic regression models were expressed as the odds ratio (OR). A p value $<0.05$ was considered statistically significant.

\section{Ethical approval}

Ethical approval was obtained from the Makerere University School of Medicine Research and Ethics Committee, the Uganda National Council for Science and Technology, the London School of Hygiene and Tropical Medicine Ethics Committee, the School of Biological and Biomedical Sciences Ethics Committee, Durham University and the University of California, San Francisco Committee on Human Research.

Table 1 Associations between measures of urbanicity and the household density of mosquitoes stratified by study site

\begin{tabular}{|c|c|c|c|c|c|c|c|c|c|c|}
\hline \multirow{2}{*}{$\begin{array}{l}\text { Urbanicity } \\
\text { metric }\end{array}$} & \multirow{2}{*}{$\begin{array}{l}\text { Exposure } \\
\text { categories }\end{array}$} & \multicolumn{3}{|l|}{ Walukuba } & \multicolumn{3}{|l|}{ Kihihi } & \multicolumn{3}{|l|}{ Nagongera } \\
\hline & & $\begin{array}{l}\text { HDM (nights } \\
\text { of collection) }\end{array}$ & $\mathrm{IRR}^{\mathbf{b}}(95 \% \mathrm{CI})$ & $\mathbf{P}$ & $\begin{array}{l}\text { HDM (nights } \\
\text { of collection) }^{a}\end{array}$ & $\mathrm{IRR}^{\mathrm{b}}(95 \% \mathrm{CI})$ & $\mathbf{P}$ & $\begin{array}{l}\text { HDM (nights } \\
\text { of collection) }^{a}\end{array}$ & $\mathrm{IRR}^{\mathrm{b}}(95 \% \mathrm{Cl})$ & $P$ \\
\hline \multirow{2}{*}{$\begin{array}{l}\text { Household } \\
\text { density }^{c}\end{array}$} & $\leq 80$ & $2.08(327)$ & $0.30(0.16-0.59)$ & $<0.001$ & 4.68 (2086) & $0.68(0.28-1.64)$ & 0.39 & $43.3(2329)$ & $\mathrm{N} / \mathrm{A}$ & \\
\hline & $>80$ & $0.90(1857)$ & & & $3.58(166)$ & & & None & & \\
\hline \multirow[t]{2}{*}{$N D V l^{d}$} & $>0.45$ & $1.85(707)$ & $0.35(0.21-0.57)$ & $<0.001$ & $4.78(1704)$ & $0.83(0.48-1.42)$ & 0.49 & $41.1(1660)$ & $1.16(0.87-1.54)$ & 0.32 \\
\hline & $\leq 0.45$ & $0.71(1477)$ & & & $4.04(548)$ & & & 48.7 (669) & & \\
\hline \multirow{2}{*}{$\begin{array}{l}\text { Night-time } \\
\text { lights }\end{array}$} & $\leq 3$ & 1.33 (1526) & $0.32(0.19-0.55)$ & $<0.001$ & $4.60(2252)$ & $\mathrm{N} / \mathrm{A}$ & & $43.3(2329)$ & N/A & \\
\hline & $>3$ & $0.49(658)$ & & & None & & & None & & \\
\hline \multirow[t]{2}{*}{ Land cover } & $\leq 20 \%$ & $1.37(1265)$ & $0.42(0.26-0.69)$ & 0.001 & Not measured & & & Not measured & & \\
\hline & $>20 \%$ & 0.68 (919) & & & & & & & & \\
\hline \multirow{2}{*}{$\begin{array}{l}\text { Composite } \\
\text { score }^{e}\end{array}$} & Low & 1.37 (1501) & $0.28(0.17-0.48)$ & $<0.001$ & $4.68(2086)$ & $0.68(0.28-1.64)$ & 0.39 & 43.3 (2329) & $\mathrm{N} / \mathrm{A}$ & \\
\hline & High & $0.44(683)$ & & & $3.58(166)$ & & & None & & \\
\hline
\end{tabular}

a Household density of mosquitoes (number of adult female anophelines caught per nights of collection)

b Incidence rate ratio

c Number of households within $100 \mathrm{~m}$ radius from participating household

d Normalized difference vegetation index

e 1 point for each individual urbanicity metric: Walukuba (low $=0-2$, high $=3-4)$, Kihihi and Nagongera $($ low $=0-1$, high $=2$ ) 
Table 2 Associations between measures of urbanicity and parasite prevalence stratified by study site

\begin{tabular}{|c|c|c|c|c|c|c|c|c|c|c|}
\hline \multirow{2}{*}{$\begin{array}{l}\text { Urbanicity } \\
\text { metric }\end{array}$} & \multirow{2}{*}{$\begin{array}{l}\text { Exposure } \\
\text { categories }\end{array}$} & \multicolumn{3}{|l|}{ Walukuba } & \multicolumn{3}{|l|}{ Kihihi } & \multicolumn{3}{|l|}{ Nagongera } \\
\hline & & $\begin{array}{l}\mathrm{PP}^{\mathrm{a}} \text { (total } \\
\text { blood smears) }\end{array}$ & $\mathrm{OR}^{\mathrm{b}}(95 \% \mathrm{Cl})$ & $\mathbf{P}$ & $\begin{array}{l}\text { PP (total blood } \\
\text { smears) }\end{array}$ & $\mathrm{OR}^{\mathrm{b}}(95 \% \mathrm{Cl})$ & $\mathbf{P}$ & $\begin{array}{l}\text { PP (total blood } \\
\text { smears) }\end{array}$ & $\mathrm{OR}^{\mathrm{b}}(95 \% \mathrm{Cl})$ & $\mathbf{P}$ \\
\hline \multirow{2}{*}{$\begin{array}{l}\text { Household } \\
\text { density }^{c}\end{array}$} & $\leq 80$ & $7.9 \%$ (406) & $0.83(0.33-2.11)$ & 0.70 & $8.4 \%$ (3151) & $0.15(0.07-0.34)$ & $<0.001$ & $22.5 \%(3231)$ & N/A & \\
\hline & $>80$ & $5.8 \%(2207)$ & & & $1.3 \%(225)$ & & & None & & \\
\hline \multirow[t]{2}{*}{$N D V l^{d}$} & $>0.45$ & $7.3 \%(854)$ & $0.67(0.33-1.34)$ & 0.26 & $8.3 \%$ (2563) & $0.76(0.34-1.67)$ & 0.49 & $23.4 \%(2247)$ & $0.87(0.63-1.20)$ & 0.40 \\
\hline & $\leq 0.45$ & $5.5 \%$ (1759) & & & $6.5 \%(813)$ & & & $20.4(984)$ & & \\
\hline \multirow{2}{*}{$\begin{array}{l}\text { Night-time } \\
\text { lights }\end{array}$} & $\leq 3$ & $6.8 \%$ (1799) & $0.72(0.34-1.51)$ & 0.38 & $7.9 \%$ (3376) & N/A & & $22.5 \%(3231)$ & N/A & \\
\hline & $>3$ & $4.6 \%(814)$ & & & None & & & None & & \\
\hline \multirow[t]{2}{*}{ Land cover } & $\leq 20 \%$ & $6.8 \%$ (1496) & $0.84(0.41-1.71)$ & 0.63 & Not measured & & & Not measured & & \\
\hline & $>20 \%$ & $5.2 \%(1117)$ & & & & & & & & \\
\hline \multirow{2}{*}{$\begin{array}{l}\text { Composite } \\
\text { score }^{\mathrm{e}}\end{array}$} & Low & $7.3 \%$ (1808) & $0.44(0.20-0.97)$ & 0.04 & $8.4 \%$ (3151) & $0.15(0.07-0.34)$ & $<0.001$ & $22.5 \%(3231)$ & N/A & \\
\hline & High & $3.4 \%(805)$ & & & $1.3 \%(225)$ & & & None & & \\
\hline
\end{tabular}

a Parasite prevalence: proportion of blood smears positive for asexual parasites

b Odds ratio adjusted for age at the time of the blood smear and repeated measures in the same household

c Number of households within $100 \mathrm{~m}$ radius from participating household

d Normalized Difference Vegetation Index

e 1 point for each individual urbanicity metric: Walukuba (low $=0-2$, high $=3-4)$, Kihihi and Nagongera $($ low $=0-1$, high $=2$ )

Table 3 Associations between measures of urbanicity and incidence of malaria stratified by study site

\begin{tabular}{|c|c|c|c|c|c|c|c|c|c|c|}
\hline \multirow{2}{*}{$\begin{array}{l}\text { Urbanicity } \\
\text { metric }\end{array}$} & \multirow{2}{*}{$\begin{array}{l}\text { Exposure } \\
\text { categories }\end{array}$} & \multicolumn{3}{|l|}{ Walukuba } & \multicolumn{3}{|l|}{ Kihihi } & \multicolumn{3}{|l|}{ Nagongera } \\
\hline & & $\begin{array}{l}\text { Malaria inci- } \\
\text { dence (PY) }\end{array}$ & $\mathrm{IRR}^{\mathrm{b}}(95 \% \mathrm{Cl})$ & $\mathbf{P}$ & $\begin{array}{l}\text { Malaria inci- } \\
\text { dence (PY) }\end{array}$ & IRR $^{\mathrm{b}}(95 \% \mathrm{Cl})$ & $\mathbf{P}$ & $\begin{array}{l}\text { Malaria inci- } \\
\text { dence (PY) }\end{array}$ & $\operatorname{IRR}^{\mathbf{b}}(95 \% \mathrm{Cl})$ & $\mathbf{P}$ \\
\hline \multirow{2}{*}{$\begin{array}{l}\text { Household } \\
\text { densityc }^{c}\end{array}$} & $\leq 80$ & $0.35(94.2)$ & $1.02(0.58-1.81)$ & 0.94 & $1.21(720.2)$ & $0.48(0.20-1.17)$ & 0.11 & $2.17(746.9)$ & N/A & \\
\hline & $>80$ & $0.36(512.5)$ & & & $0.60(51.6)$ & & & None & & \\
\hline \multirow[t]{2}{*}{$N D V I^{d}$} & $>0.45$ & $0.43(197.6)$ & $0.77(0.46-1.28)$ & 0.31 & $1.19(586.4)$ & $0.97(0.64-1.45)$ & 0.87 & $1.91(519.6)$ & $1.35(1.07-1.70)$ & 0.01 \\
\hline & $\leq 0.45$ & $0.32(409.1)$ & & & $1.11(185.3)$ & & & $2.75(227.3)$ & & \\
\hline \multirow{2}{*}{$\begin{array}{l}\text { Night-time } \\
\text { lights }\end{array}$} & $\leq 3$ & $0.36(418.3)$ & $1.04(0.62-1.75)$ & 0.88 & $1.17(771.8)$ & N/A & & $2.17(746.9)$ & N/A & \\
\hline & $>3$ & $0.36(188.4)$ & & & None & & & None & & \\
\hline \multirow[t]{2}{*}{ Land cover } & $\leq 20 \%$ & $0.37(346.6)$ & $1.04(0.62-1.73)$ & 0.89 & Not measured & & & Not measured & & \\
\hline & $>20 \%$ & $0.35(260.1)$ & & & & & & & & \\
\hline \multirow{2}{*}{$\begin{array}{l}\text { Composite } \\
\text { score }^{\mathrm{e}}\end{array}$} & Low & 0.37 (419.0) & $0.97(0.57-1.65)$ & 0.92 & $1.21(720.2)$ & $0.48(0.20-1.17)$ & 0.11 & $2.17(746.9)$ & N/A & \\
\hline & High & $0.34(187.7)$ & & & $0.60(51.6)$ & & & None & & \\
\hline
\end{tabular}

${ }^{a}$ Number of episodes of malaria per person years (PY) of follow-up

${ }^{\mathrm{b}}$ Incidence rate ratio adjusted for mean age during follow-up and repeated measures in the same household

c Number of households within $100 \mathrm{~m}$ radius from a participating household

${ }^{d}$ Normalized difference vegetation index

e 1 point for each individual urbanicity metric: Walukuba (low $=0-2$, high $=3-4)$, Kihihi and Nagongera (low $=0-1$, high $=2$ )

\section{Results}

\section{Study population}

A total of 1167 participants (878 children and 289 adults) in 300 households were enrolled and followed over the 2-year period; 348 in Walukuba, 419 in Kihihi and 400 in Nagongera. Overall, 1087 participants $(93.1 \%)$ and 292 households (97.3\%) were followed for at least 6 months. Characteristics of study households and cohort participants are presented in Table 4. Households in Walukuba were much more characteristic of an urban environment compared to the two rural sites (Kihihi and Nagongera), including a higher proportion with electricity ( 22 vs $3 \%, \mathrm{p}<0.001)$ and the use of charcoal versus firewood for cooking (82 vs $5 \%, \mathrm{p}<0.001$ ), a higher density of surrounding households (225 vs 13 within a $100 \mathrm{~m}$ radius, $\mathrm{p}<0.001)$, a lower vegetation index (66 
Table 4 Characteristics of study households and participants stratified by study site

\begin{tabular}{|c|c|c|c|}
\hline Characteristics & Walukuba & Kihihi & Nagongera \\
\hline \multicolumn{4}{|l|}{ At the household level } \\
\hline Number of households & 100 & 100 & 100 \\
\hline Average altitude (m) & 1165 & 1103 & 1130 \\
\hline Electricity in home & $22 \%$ & $4 \%$ & $1 \%$ \\
\hline Uses charcoal for cooking & $82 \%$ & $10 \%$ & $0 \%$ \\
\hline Density of surrounding households ${ }^{\mathrm{a}}$, mean (SD) & $225(152)$ & $19(32)$ & $7(6)$ \\
\hline NDVI $<0.45$ units $^{\mathrm{a}}$ & $66 \%$ & $26 \%$ & $28 \%$ \\
\hline Night-time lights $>3$ units $^{\mathrm{a}}$ & $29 \%$ & $0 \%$ & $0 \%$ \\
\hline Mean land cover $>20 \%$ residential urban ${ }^{a}$ & $41 \%$ & $\mathrm{~N} / \mathrm{A}$ & $\mathrm{N} / \mathrm{A}$ \\
\hline Total number of nights of mosquito collections & 2184 & 2252 & 2329 \\
\hline Total number of female Anopheles mosquitoes & 2358 & 10,370 & 100,890 \\
\hline Household density of mosquitoes per night (95 \% Cl) & $1.1(1.0-1.1)$ & $4.6(4.5-4.7)$ & $43.3(43.1-43.6)$ \\
\hline \multicolumn{4}{|l|}{ At the individual cohort participant level } \\
\hline Number of participants & 348 & 419 & 400 \\
\hline Mean age in years during follow-up (children) & 5.0 & 5.6 & 5.4 \\
\hline Mean age in years during follow-up (adults) & 33.4 & 38.3 & 39.0 \\
\hline Total number of routine blood slides & 2613 & 3376 & 3231 \\
\hline Parasite prevalence (95 \% Cl) & $6.1(5.2-7.1 \%)$ & $7.9(7.0-8.8 \%)$ & $22.5(21.0-23.9 \%)$ \\
\hline Person years of observation & 606.7 & 771.8 & 746.9 \\
\hline Total episodes of malaria & 217 & 905 & 1619 \\
\hline Incidence of malaria per person years (95 \% Cl) & $0.36(0.31-0.41)$ & $1.17(1.10-1.25)$ & $2.17(2.06-2.28)$ \\
\hline
\end{tabular}

${ }^{a}$ Within $100 \mathrm{~m}$ radius of study household

vs $27 \%$ with NDVI $<0.45$ units, $\mathrm{p}<0.001)$, and more night-time lights ( 29 vs $0 \%$ with $>3$ units, $\mathrm{p}<0.001)$. In addition, the number of female Anopheles mosquitoes captured per night was significantly lower in Walukuba $(\mathrm{HDM}=1.1)$ compared to Kihihi $(\mathrm{HDM}=4.6, \mathrm{p}<0.001)$ and Nagongera (HDM $=43.3, \mathrm{p}<0.001)$. At the level of the individual cohort participants, parasite prevalence in Walukuba (6.1 \%) was similar to Kihihi (7.9 \%, $\mathrm{p}=0.14$ ) but significantly lower than in Nagongera $(22.5 \%, \mathrm{p}<0.001)$. The incidence of malaria in Walukuba (0.36 episodes per person year (PY)) was significantly lower than in Kihihi $(1.17$ episodes PY, p < 0.001) and Nagongera (2.17 episodes PY, $\mathrm{p}<0.001)$.

\section{Mosquito species composition}

The totals of female Anopheles collected over the 24-month period were 2358 in Walukuba, 10,370 in Kihihi and 100,797 in Nagongera. The most common species was Anopheles gambiae, with $86.3 \%$ of those caught in Walukuba, $99.0 \%$ in Kihihi and $90.3 \%$ in Nagongera. Anopheles funestus, made up $6.7 \%$ of catches in Walukuba, 0.0 \% in Kanungu and $9.1 \%$ in Nagongera. Finally, other female Anopheles species caught together made up $7.0 \%$ of catches in Walukuba, $1.0 \%$ in Kihihi and $0.7 \%$ in Nagongera.

\section{Associations between measures of urbanicity and the household density of mosquitoes}

Within the more urban site of Walukuba, individual households with characteristics of greater urbanicity were associated with a lower HDM including a household density $>80$ per $100 \mathrm{~m}$ radius (IRR $=0.30, \mathrm{p}<0.001$ ), NDVI $\leq 0.45$ (IRR $=0.35, \mathrm{p}<0.001)$, night-time lights $>3$ units $(\mathrm{IRR}=0.32, \mathrm{p}<0.001)$, and land cover $>20 \%$ $(\mathrm{IRR}=0.42, \mathrm{p}<0.001)$ (Table 1$)$. In contrast, within the two rural sites, individual households with characteristics of greater urbanicity were either not significantly associated with HDM (household density or NDVI), outside the range where associations were found in Walukuba (night-time lights) or lacked sufficient variability to be measured (land cover) (Table 1). Within Walukuba, three geographical clusters of households with distinct characteristics were observed (Fig. 3), including: (1) a fishing village comprised of make-shift wooden housing; (2) slam of predominantly mud and wattle house structures mixed with sub-urban household structures randomly situated without obvious general urban planning; and, (3) old, but fairly orderly, factory workers' estate housing. Across these clusters, the totals of female Anopheles collected over the 24-month period were 1256 (53.3\%), 666 (28.2\%) and 436 (18.5\%), respectively. 


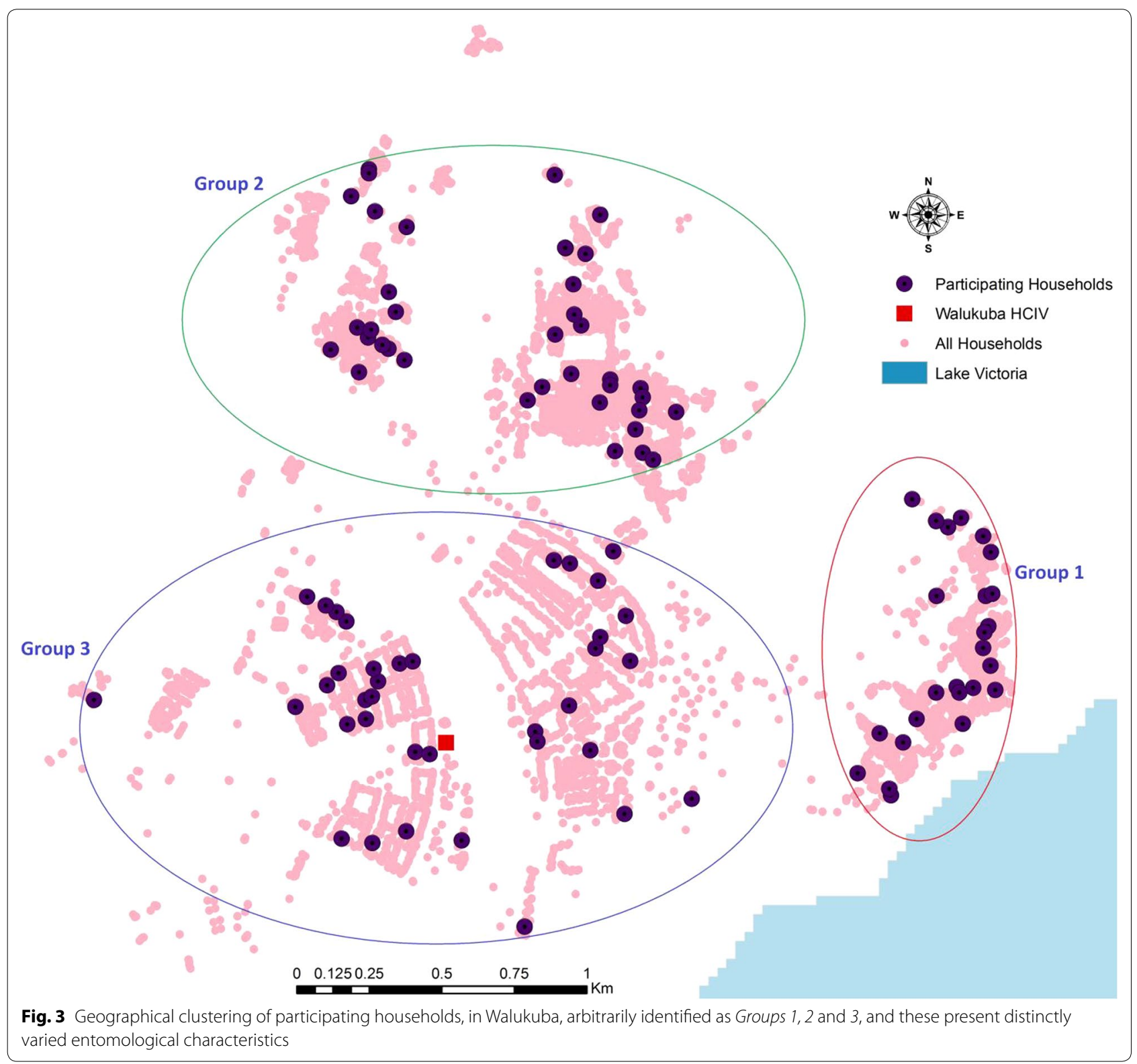

\section{Associations between measures of urbanicity and parasite} prevalence

In Walukuba (more urban site), individuals living in households with characteristics of greater urbanicity were associated with lower parasite prevalence, but none of these associations reached statistical significance (Table 2). However, a higher composite score was associated with a significantly lower parasite prevalence $(\mathrm{OR}=0.44, \mathrm{p}=0.04)$. In the rural site of Kihihi, individuals living in households with a household density $>80$ per $100 \mathrm{~m}$ radius were associated with a lower parasite prevalence $(\mathrm{OR}=0.15, \mathrm{p}<0.001)$. In the most rural site of Nagongera, only individuals living in households with an NDVI $<0.45$ could be evaluated and this measure of urbanicity was not significantly associated with parasite prevalence (Table 2).

\section{Associations between measures of urbanicity and incidence of malaria}

None of the measures of urbanicity, either individually or as a composite score, were associated with a lower incidence of malaria in individual study participants at any of the three sites (Table 3). In Nagongera, however, living in households with an NDVI $<0.45$ was associated with a higher incidence of malaria $(\mathrm{IRR}=1.35$, $\mathrm{p}=0.01$ ). 


\section{Discussion}

Evidence for the impact of urbanicity on reduced malaria prevalence over large scales is clear $[3,8]$ but the interplay between local heterogeneities, differing malaria metrics and different metrics of urbanicity has rarely been explored. Here, these relationships have been assessed for three sites in Uganda with markedly differing malaria ecologies. The data presented show that variations in the HDM, parasite prevalence and the incidence of malaria in the three sub-counties of Uganda correspond to relative measures of urbanicity across the three sites. Within each site, associations were significant between greater urbanicity and a lower HDM and parasite prevalence in the most urban site (Walukuba), but less so for the two rural sites. Moreover, different approaches to measuring urbanicity translated into slightly different relationships with malaria-relevant metrics, indicating that each urbanicity measure captures different aspects that may or may not be driving mosquito and malaria dynamics.

Increased levels of urbanicity, specifically defined by higher household density, lower levels of NDVI, low residential urban land cover and higher night-time light brightness were observed to be strongly associated with reduced HDM at the urban site, Walukuba, but not in the other two more rural sites. For Walukuba, parasite prevalence was not found to be significantly associated with individual urbanicity metrics at the spatial scales examined here, but rather with the composite urbanicity score. However, increased urbanicity as measured by household density does appear to be associated with reduced parasite prevalence in one rural site, Kihihi, but does not in the other.

Additional observations of disease burden across the three sites indicate that the urban site recorded significant declines in malaria incidence over the study duration [16]. With the observed consistent associations between some but not all urban metrics and malaria metrics, the principal group of urban metrics that showed the most consistent associations with reduced malaria metrics included high household density, low NDVI, high night-time light brightness, and low dense urban land cover. These may be good candidates for future use in modelling the impact of urbanicity on malaria, and wider malaria risk mapping in general.

Whereas higher parasite prevalence was found in the more rural sites than Walukuba, consistent with multiple studies that have reported similar trends $[8,24]$, only household density had significant associations with it in the rural sites. Unlike this study, other studies have reported significant associations between parasite prevalence and urbanization at sub-continental and global scales $[2,3]$. These survey data, however, unlike cohort data, tend to go through long compilation processes, come from various sources collected in differing seasons and often with overlapping age groups [25].

Some aspects of this study limit the ability to draw additional conclusions about urbanicity and malaria transmission. With just 100 households per site, sufficient variability may not have been captured to uncover weaker relationships that may have existed, especially within the more rural sites. These households were, however, randomly selected without bias and are thus likely representative of the populations, and the observational buffers used describe an environment shared by a larger number of households. Another notable limitation is that Nagongera and Kihihi are not so different or diverse in terms of urbanicity, and neither can be described as particularly 'urban' overall. High-resolution imagery that could better elicit differences between these sites is not readily available. However, malaria metrics between the two are substantially different and hence do provide a useful contrast in transmission. Factors such as agricultural practices may influence the malaria epidemiology of these rural regions far more than any urban-related factors, but analysis of these were beyond the scope of this current paper.

Other measures used to define urbanicity that were not assessed in this study include the principal economic activity of the population. However, in a study of the risk of malaria in these three sites posed by the nature of house of residence, strong associations were found between HDM and type of house or wealth [26]. In further regard to the measures of urbanization, it was not clear what other factor(s) of urbanization are the most relevant for malaria as a benchmark for the expected outcome to set the stage for auxiliary exploratory work. In addition, this study did not account for contextual factors that may be at play in the study sites, such as coverage of malaria control interventions, multiple interventions' interference and/or influence, climate and climate change, or variability as played out by political and other forces of influence between jurisdictions of the study sites, to mention just a few. Finally, despite the length of follow-up of the cohorts, in this study temporal variations in urban growth versus malaria were not examined.

In view of these limitations, future work may focus on better understanding the impact of urbanization on malaria through expanded analyses to investigate the role played by other contributory factors, such as rainfall, water bodies, temperature, intervention coverage, as well as nuances of malaria policy disparities across administrative units. Considering that other than space, the temporal aspects of public health are important, additional work should examine the impact of the temporal variations in urbanicity and other factors on malaria. Also, 
given a stronger understanding of the urbanicity influence on malaria, scaling up analyses to generate local and national risk maps would be a valuable effort for guiding malaria and other public health strategic planning. This, with the integration of mathematical transmission models to bolster guidance for planning for public health and malaria control, would be an important next step. Finally, given previous studies on travel and movement patterns in East Africa, and their perceived, as well as reported correlation with urbanization, proposed future work may incorporate these influences to further establish the contribution of travel and movement coupled with urbanization, to the malaria situation.

Given differences in culture/behaviour, population characteristics, socio-economic strata, and environment between urban and rural communities, these results, though exploratory in nature, support the need for more localized approaches to malaria control and treatment, such as urbanicity tailored packaging of strategic planning foci, intervention approaches, impact evaluation, malaria awareness messages and/or public health campaigns. Africa, more than ever, is becoming increasingly urbanized and targeted interventions such as better garbage and drainage management coupled with LSM in urban areas, would likely results in a great depletion of breeding sites. Given the burden of malaria in sub-Saharan Africa, there is a need to understand the broad impacts of urbanicity on malaria at various scales. Whereas previous studies have quantified these impacts chiefly on large scales and used just single metrics for quantifying the relationship between urbanicity and malaria, this study has shown that the situation is much more complex at smaller scales.

\section{Abbreviations}

IRR: incidence rate ratio; $\mathrm{Cl}$ : 95 \% confidence interval; OR: odds ratio; NDVI: normalized difference vegetation index; LLINs: long-lasting insecticidal nets; IRS: indoor residual spraying; IVM: integrated vector management; LSM: laval source management; GPS: global positioning system; CDC: Centers for Disease Control; HDM: household density of mosquitoes; ETM: enhanced thematic map; VIIRS: visible infrared imaging radiometer suite; PY: per person year.

\section{Authors' contributions}

MRK, SGS, SL, DLS, AJT and GD conceived and designed the study. SPK, EA, AK, MK, JN, SGS, SL, GD, MRK, and AJT participated in the data collection. SPK, DP, GD and AJT participated in the management and analysis of the data. All authors participated in the writing of the manuscript. All authors read and approved the final manuscript.

\footnotetext{
Author details

${ }^{1}$ Infectious Diseases Research Collaboration, Kampala, Uganda. ${ }^{2}$ Clinton Health Access Initiative, Boston, USA. ${ }^{3}$ Fogarty International Center, National Institutes of Health, Bethesda, USA. ${ }^{4}$ Department of Zoology, University of Oxford, Oxford, UK. ${ }^{5}$ Sanaria Institute for Global Health and Tropical Medicine, Rockville, MD, USA. ${ }^{6}$ School of Biological and Biomedical Sciences, Durham University, Durham, UK. ${ }^{7}$ London School of Hygiene and Tropical Medicine, London, UK. ${ }^{8}$ Department of Medicine, San Francisco General Hospital, University of California, San Francisco, CA, USA. ${ }^{9}$ School of Medicine,
}

Makerere University College of Health Sciences, Kampala, Uganda. ${ }^{10}$ Department of Geography and Environment, University of Southampton, Southampton, UK. ${ }^{11}$ Flowminder Foundation, Stockholm, Sweden.

\section{Acknowledgements}

This study was realized through the help of research Funders, cooperative study participants and their families, the Makerere University-UCSF Research Collaboration and Infectious Diseases Research Collaboration (IDRC) with administrative and technical support, and a hard working study team. Funding was provided by the National Institutes of Health as part of the International Centers of Excellence in Malaria Research (ICMER) (U19AI089674). AJT and DLS acknowledge funding support from the RAPIDD of the Science and Technology Directorate, Department of Homeland Security, and the Fogarty International Center, National Institutes of Health. AJT is also supported by grants from the Bill and Melinda Gates Foundation (OPP1 106427and \#1032350). DLS acknowledges funding from the Bill \& Melinda Gates Foundation (\#OPP1110495). The funders had no role in the study design, data collection and analysis, decision to publish or preparation of the manuscript.

\section{Compliance with ethical guidelines}

\section{Competing interests}

The authors declare that they have no competing interests.

Received: 14 February 2015 Accepted: 22 August 2015

Published online: 29 September 2015

\section{References}

1. De Silva PM, Marshall JM. Factors contributing to urban malaria transmission in sub-saharan Africa: a systematic review. J Trop Med. 2012;2012:819563.

2. Hay SI, Guerra CA, Tatem AJ, Atkinson PM, Snow RW. Urbanization, malaria transmission and disease burden in Africa. Nat Rev Microbiol. 2005;3:81-90

3. Qi Q, Guerra CA, Moyes CL, Elyazar IR, Gething PW, Hay SI, et al. The effects of urbanization on global Plasmodium vivax malaria transmission. Malar J. 2012;11:403.

4. Tatem AJ, Gething PW, Smith DL, Hay SI. Urbanization and the global malaria recession. Malar J. 2013;12:133.

5. Tatem AJ, Guerra CA, Kabaria CW, Noor AM, Hay SI. Human population, urban settlement patterns and their impact on Plasmodium falciparum malaria endemicity. Malar J. 2008;7:218.

6. Dye C. Health and urban living. Science. 2008;319:766-9.

7. Utzinger J, Keiser J. Urbanization and tropical health: then and now. Ann Trop Med Parasitol. 2006;100:517-33.

8. Pond BS. Malaria indicator surveys demonstrate a markedly lower prevalence of malaria in large cities of sub-Saharan Africa. Malar J. 2013;12:313.

9. Matthys B, Vounatsou P, Raso G, Tschannen AB, Becket EG, Gosoniu L, et al. Urban farming and malaria risk factors in a medium-sized town in Cote d'Ivoire. Am J Trop Med Hyg. 2006;75:1223-31.

10. Talisuna A, Adibaku S, Dorsey G, Kamya MR, Rosenthal PJ. Malaria in Uganda: challenges to control on the long road to elimination II. The path forward. Acta Trop. 2012;121:196-201.

11. Gething PW, Patil AP, Smith DL, Guerra CA, Elyazar IR, Johnston GL, Tatem AJ, Hay SI. A new world malaria map: Plasmodium falciparum endemicity in 2010. Malar J. 2011;10:378.

12. Okiro EA, Bitira D, Mbabazi G, Mpimbaza A, Alegana VA, Talisuna AO, et al. Increasing malaria hospital admissions in Uganda between 1999 and 2009. BMC Med. 2011;9:37.

13. Yeka A, Gasasira A, Mpimbaza A, Achan J, Nankabirwa J, Nsobya S, et al. Malaria in Uganda: challenges to control on the long road to elimination: I. Epidemiology and current control efforts. Acta Trop. 2012;121:184-95.

14. WHO. Larval source management: a supplementary malaria vector control measure: an operational manual. Geneva: World Health Organization; 2013

15. Staedke SG. Phase 1 Report: Tororo District Survey Project. Characterizing the population and local health services. 2010. 
16. Kamya MR, Arinaitwe E, Wanzira H, Katureebe A, Barusya C, Kigozi SP, et al. Malaria transmission, infection, and disease at three sites with varied transmission intensity in Uganda: implications for malaria control. Am J Trop Med Hyg. 2015;92:903-12.

17. Kilama M, Smith DL, Hutchinson R, Kigozi R, Yeka A, Lavoy G, et al. Estimating the annual entomological inoculation rate for Plasmodium falciparum transmitted by Anopheles gambiae s.l. using three sampling methods in three sites in Uganda. Malar J. 2014;13:111.

18. Google Earth [http://www.earth.google.com]. Accessed 16 May 2013.

19. USGS Science for a changing world [http://www.glovis.usgs.gov/]. Accessed 5 Nov 2013.

20. Tatem AJ, Noor AM, von Hagen C, Di Gregorio A, Hay SI. High resolution population maps for low income nations: combining land cover and census in East Africa. PLoS One. 2007;2:e1298.

21. Weier J, Herring D. Measuring vegetation (ndvi \& evi). NASA online publication. 2011.

22. Keiser J, Utzinger J, Caldas de Castro M, Smith TA, Tanner M, Singer BH. Urbanization in sub-saharan Africa and implication for malaria control. Am J Trop Med Hyg. 2004;71:118-27.
23. Xie PJ, Tan ZF, Hou JC, Wang MB. Analysis on dynamic relationship between urbanization and electricity consumption level in China. Power Syst Technol. 2009;33:77.

24. Robert V, Macintyre K, Keating J, Trape JF, Duchemin JB, Warren M, Beier JC. Malaria transmission in urban sub-Saharan Africa. Am J Trop Med Hyg. 2003;68:169-76.

25. Gemperli A, Sogoba N, Fondjo E, Mabaso M, Bagayoko M, Briet OJ, Anderegg D, Liebe J, Smith T, Vounatsou P. Mapping malaria transmission in West and Central Africa. Trop Med Int Health. 2006;11:1032-46.

26. Wanzirah H, Tusting LS, Arinaitwe E, Katureebe A, Maxwell K, Rek J, Bottomley C, Staedke SG, Kamya M, Dorsey G, Lindsay SW. Mind the gap: house structure and the risk of malaria in Uganda. PLoS One. 2015;10:e0117396.

\section{Submit your next manuscript to BioMed Central and take full advantage of:}

- Convenient online submission

- Thorough peer review

- No space constraints or color figure charges

- Immediate publication on acceptance

- Inclusion in PubMed, CAS, Scopus and Google Scholar

- Research which is freely available for redistribution

Submit your manuscript at

www.biomedcentral.com/submit

C BioMed Central 